\title{
Comparison of Sensitivities of American College of Rheumatology and Systemic Lupus International Collaborating Clinics Classification Criteria in Childhood-onset Systemic Lupus Erythematosus
}

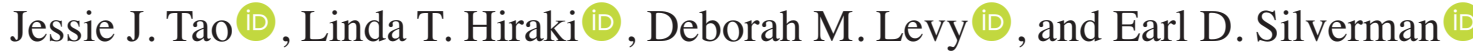

ABSTRACT. Objective. Currently there are 2 different classification criteria for systemic lupus erythematosus (SLE): American College of Rheumatology (ACR) and Systemic Lupus International Collaborating Clinics (SLICC). The aim of this study was to compare the sensitivities of ACR and SLICC criteria in childhood-onset SLE (cSLE) using a large, multiethnic cohort.

Methods. We conducted a retrospective study of 722 patients diagnosed with cSLE at The Hospital for Sick Children (SickKids). Prospectively collected data from SickKids' Lupus Database were reviewed/validated against medical records prior to ACR and SLICC scoring based on cumulative symptoms up to the last visit. Sensitivities were compared using McNemar's test. Descriptive statistics were used to identify SLE features unique to each set of criteria and autoantibodies not included in either.

Results. ACR and SLICC sensitivities were as follows: $92.4 \%$ and $96.3 \%$ overall $(\mathrm{p}=0.001) ; 82.5 \%$ and $91.3 \%(\mathrm{p}=0.01)$ in those scored $\leq 1$ year from diagnosis; $92.7 \%$ and $97.9 \%(\mathrm{p}=0.02)$ in those scored 2-3 years from diagnosis. Forty-eight of $55(87.3 \%)$ patients who did not meet ACR criteria met SLICC criteria through SLICC-specific criterion or renal biopsy. Twenty of $27(74.1 \%)$ patients who did not meet SLICC criteria met ACR criteria as a result of photosensitivity (73.9\%) and ACR lymphopenia criteria (26.1\%). Six of 7 patients $(85.7 \%)$ who were clinically diagnosed with cSLE but did not meet either SLICC or ACR criteria had anti-Ro antibodies.

Conclusion. SLICC criteria were significantly more sensitive than ACR criteria in cSLE classification, especially early in the disease course. Because of the extreme rarity of primary Sjögren syndrome in children, one may consider adding anti-Ro antibodies to the classification criteria for cSLE because they are present in $\sim 40 \%$ of patents with cSLE. (First Release February 15 2019; J Rheumatol 2019;46:731-8; doi:10.3899/jrheum.180337)

Key Indexing Terms:

SYSTEMIC LUPUS ERYTHEMATOSUS AMERICAN COLLEGE OF RHEUMATOLOGY

SYSTEMIC LUPUS INTERNATIONAL COLLABORATING CLINICS

CLASSIFICATION CRITERIA CHILDHOOD-ONSET SYSTEMIC LUPUS ERYTHEMATOSUS

Systemic lupus erythematosus (SLE) is a multisystem autoimmune disease that mainly affects women of child-

From the Division of Rheumatology, The Hospital for Sick Children; Faculty of Medicine, University of Toronto; Translational Medicine, SickKids Research Institute, Toronto, Ontario, Canada.

J.J.Tao, BSc, Division of Rheumatology, The Hospital for Sick Children, and Faculty of Medicine, University of Toronto, and Translational Medicine, SickKids Research Institute; L.T. Hiraki, MD, FRCPC, ScD, Division of Rheumatology, The Hospital for Sick Children, and Faculty of Medicine, University of Toronto, and Translational Medicine, SickKids Research Institute; D.M. Levy, MD, FRCPC, Division of Rheumatology, The Hospital for Sick Children, and Faculty of Medicine, University of Toronto, and Translational Medicine, SickKids Research Institute; E.D. Silverman, MD, FRCPC, Division of Rheumatology, The Hospital for Sick Children, and Faculty of Medicine, University of Toronto, and Translational Medicine, SickKids Research Institute.

Address correspondence to Dr. E.D. Silverman, The Hospital for Sick Children, 555 University Ave., Toronto, Ontario M5G 1X8, Canada.

E-mail: earl.silverman@sickkids.ca

Accepted for publication October 4, 2018. bearing age, with about $20 \%$ beginning in childhood and adolescence. Patients with SLE vary greatly in clinical and laboratory presentation and may be confused with patients who have other systemic autoimmune diseases. Therefore in 1971, the American College of Rheumatology (ACR) developed a set of classification criteria that had the best sensitivity and specificity to collect a well-defined SLE research patient population that can be objectively differentiated from other autoimmune diseases ${ }^{1}$. The ACR criteria have since been modified from the 1971 criteria: once in 1982 to improve its sensitivity and specificity and again in 1997 to reflect new antiphospholipid antibody testing 2,3 .

As early as 2003, the Systemic Lupus International Collaborating Clinics (SLICC) group realized that the 1997 criteria may need further revision ${ }^{4}$. In 2012, new SLICC classification criteria were published for $\mathrm{SLE}^{5}$. The new SLICC criteria had increased the sensitivity of ACR criteria

Personal non-commercial use only. The Journal of Rheumatology Copyright @ 2019 . All rights reserved. 
from $83 \%$ to $97 \%$ but decreased the specificity from $96 \%$ to $84 \%$. Overall, it resulted in a lower number of misclassified cases $^{5}$.

Although both ACR and SLICC criteria are used in childhood-onset SLE (cSLE) research, both these criteria sets have only been validated by a few studies in cSLE, all with relatively small sample sizes. To date, there have been only 2 studies comparing the sensitivity and specificity of ACR and SLICC criteria in $\mathrm{CSLE}^{6,7}$. More recently, there has been a larger study examining only the sensitivity of the 2 classification criteria ${ }^{8}$. The aim of our study was to determine and compare the sensitivities of ACR and SLICC criteria in cSLE within a large, multiethnic, single-center cohort. Secondary aims were to identify the frequency of the specific features that resulted in patients meeting only one of the 2 classification criteria sets, to identify any autoantibodies seen in patients with cSLE that were not recorded in either set of criteria, and to assess sensitivities of criteria across sexes, ethnicities, and time from diagnosis.

\section{MATERIALS AND METHODS}

Study population. The SickKids Lupus Database was queried for all patients entered between January 1984 and May 2017 and 758 patients met the entry criteria: (1) diagnosed and followed at SickKids Hospital; and (2) diagnosed with CSLE (defined as diagnosis prior to age 18) or diagnosed with incipient cSLE (ISLE; did not meet ACR or SLICC criteria for SLE but had clinical and laboratory features suggestive of SLE). The SickKids Lupus Database has prospectively collected all clinical and laboratory data on each patient since its inception in January 1984 using a structured data collection form. Of the 758 patients, 722 (95\%) were diagnosed with cSLE and $36(5 \%)$ had ISLE. All diagnoses, including ACR-/SLICC-patients, had $100 \%$ agreement of all 3 expert pediatric staff rheumatologists (EDS, DML, LTH with 37, 15, and 10 yrs of experience treating patients with cSLE, respectively). Patients with ISLE met neither expert opinion nor ACR or SLICC criteria. The final study cohort therefore consisted of 722 patients with cSLE.

This study has been approved by REB at SickKids Hospital (REB no. 1000028143).

Data collection. Baseline features and any new feature(s) seen at each visit were recorded and entered prospectively into the database. The SickKids Lupus Database has prospectively collected all clinical and laboratory data on each patient since its inception in January 1984 using a structured data collection form. All patient information within the SickKids Lupus Database was reviewed and then validated against medical records prior to the validation of ACR and SLICC classification criteria scores. These classifications were based on cumulative symptoms up to the time of the last visit ${ }^{5}$. Following this, all patients were then grouped into 4 mutually exclusive categories based on their fulfillment of the following: (1) Both ACR and SLICC classification criteria (ACR+/SLICC+); (2) ACR but not SLICC classification criteria (ACR+/SLICC-); (3) SLICC but not ACR classification criteria (ACR-/SLICC+); or (4) neither ACR or SLICC classification criteria but diagnosis based on expert opinion (ACR-/SLICC-). For ACR-/SLICC- patients, the reason for diagnosis of cSLE was noted.

Data analysis. We used descriptive statistics to describe our cohort. Additionally, the sensitivity of each criteria set was calculated and then compared to each other using McNemar's test because both criteria were applied to each patient, resulting in paired nominal data. The level of significance was set at $\mathrm{p}<0.05$

All analyses were done using JASP (version 0.8.1.2) and Microsoft Excel (version 14.7.2).

\section{RESULTS}

Demographics. Our study cohort consisted of 722 patients diagnosed with cSLE: 590 (81.7\%) were female with a mean age of diagnosis of 12.8 years \pm 3 years. The predominant ethnicities in our cohort were Asian (36.6\%), white (29.2\%), black (14.0\%), and Hispanic (3.9\%). In addition, 5.3\% identified as mixed and $10.4 \%$ were of unknown ethnicity (Table 1).

Comparison of ACR and SLICC classification sensitivity. There was a greater number of patients who had $\geq 4$ SLICC classification criteria [695/722 patients $(96.3 \%)]$ than who met $\geq 4$ ACR classification criteria [667/722 patients $(92.4 \%) ; \mathrm{p}=0.001]$. The mean number of SLICC classification criteria met by our total cohort was $7.5( \pm$ SD 2.5$)$ with a range of $2-15$ criteria, while the mean number of ACR criteria was $5.7( \pm$ SD 1.5) with a range of 1-10 criteria (Figure 1). When we excluded the 7 patients who were diagnosed by expert opinion only, the mean number of SLICC classification criteria at $7.5( \pm$ SD 2.5$)$ and the mean number of ACR classification at 5.7 ( \pm SD 1.5) did not change, but the range of values decreased to $3-15$ criteria and 2-10 criteria, respectively.

Effect of sex. Of the females, 548/590 (92.9\%) met ACR classification criteria, whereas 567/590 (96.1\%) met SLICC classification criteria $(\mathrm{p}=0.02)$. Similarly, among the males, 119/132 (90.2\%) met ACR classification criteria and 128 (97.0\%) met SLICC classification criteria $(\mathrm{p}=0.02)$.

Effect of ethnicity. There were a greater number of patients who met SLICC classification criteria $(259 / 264,98.1 \%)$ compared to ACR classification criteria (248/264, 93.9\%; $\mathrm{p}=0.006)$ in the Asian subgroup. Although non-statistically significant, similar findings were noted across white, black, Hispanic, mixed, and unknown ethnic subgroups (Table 1).

Effect of time of scoring from diagnosis. The study cohort was scored according to both ACR and SLICC classification criteria based on all SLE features present up until their most recent visit. There were $183(25.3 \%)$ scored within $\leq 1$ year, $192(26.6 \%)$ scored between 2-3 years, 133 (18.4\%) scored within 4-5 years, $145(20.1 \%)$ scored within 6-10 years, and $69(9.6 \%)$ scored beyond 10 years of initial diagnosis. The sensitivities of both ACR and SLICC classification criteria followed an upward trend as time progressed from the initial diagnosis: $82.5 \%$ versus $91.3 \%(\mathrm{p}=0.01)$ at $\leq 1$ year, $92.7 \%$ versus $97.9 \%(\mathrm{p}=0.02)$ at $2-3$ years, $95.5 \%$ versus $98.5 \%$ $(\mathrm{p}=0.5)$ at $4-5$ years, $97.9 \%$ versus $96.6 \%(\mathrm{p}=0.7)$ at $6-10$ years, and $100.0 \%$ versus $100.0 \%$ at $>10$ years.

The cohort was then divided into 4 mutually exclusive categories: ACR+/SLICC+, ACR-/SLICC+, ACR+/SLICC-, and ACR-/SLICC- (Table 2).

(1) Six hundred forty-seven of the 722 (89.6\%) patients met both ACR and SLICC classification criteria (ACR+/SLICC+; Table 2).

(2) Forty-eight patients $(6.6 \%)$ had $<4$ ACR classification

Personal non-commercial use only. The Journal of Rheumatology Copyright @ 2019. All rights reserved. 
Table 1. Summary of ACR and SLICC sensitivity.

\begin{tabular}{|c|c|c|c|c|c|c|}
\hline \multirow[t]{2}{*}{ Variables } & \multirow[t]{2}{*}{ Total, n (\%) } & \multicolumn{2}{|c|}{ ACR } & \multicolumn{2}{|c|}{ SLICC } & \multirow[b]{2}{*}{$\mathrm{p}$} \\
\hline & & ${ }^{ \pm}$Positive, $\mathrm{n}$ & Sensitivity, $\%$ & ${ }^{ \pm \pm}$Positive, $\mathrm{n}$ & Sensitivity, $\%$ & \\
\hline All patients & $722(100)$ & 667 & 92.4 & 695 & 96.3 & $0.001 *$ \\
\hline Female & $590(81.7)$ & 548 & 92.9 & 567 & 96.1 & $0.02 *$ \\
\hline Male & $132(18.3)$ & 119 & 90.2 & 128 & 97.0 & $0.02 *$ \\
\hline \multicolumn{7}{|l|}{ Ethnicity } \\
\hline Black & $101(14.0)$ & 96 & 95.0 & 99 & 98.0 & 0.4 \\
\hline Hispanic & $28(3.9)$ & 26 & 92.9 & 27 & 96.4 & 1 \\
\hline Aboriginal & $4(0.6)$ & 4 & 100.0 & 4 & 100.0 & - \\
\hline Pacific Islander & $1(0.1)$ & 1 & 100.0 & 1 & 100.0 & - \\
\hline Mixed & $38(5.3)$ & 36 & 94.7 & 38 & 100.0 & 0.5 \\
\hline Unknown & $75(10.4)$ & 61 & 81.3 & 67 & 89.3 & 0.3 \\
\hline $6-10 \mathrm{yrs}$ & $145(20.1)$ & 142 & 97.9 & 140 & 96.6 & 0.7 \\
\hline$>10 \mathrm{yrs}$ & $69(9.6)$ & 69 & 100.0 & 69 & 100.0 & - \\
\hline
\end{tabular}

* $\mathrm{P}<0.05$ indicates a statistically significant difference between the sensitivity of ACR and SLICC classification criteria. ${ }^{ \pm}$Individuals who met $\geq 4$ ACR classification criteria. ${ }^{ \pm \pm}$Individuals who met $\geq 4$ SLICC classification criteria. ACR: American College of Rheumatology; SLICC: Systemic Lupus International Collaborating Clinics.

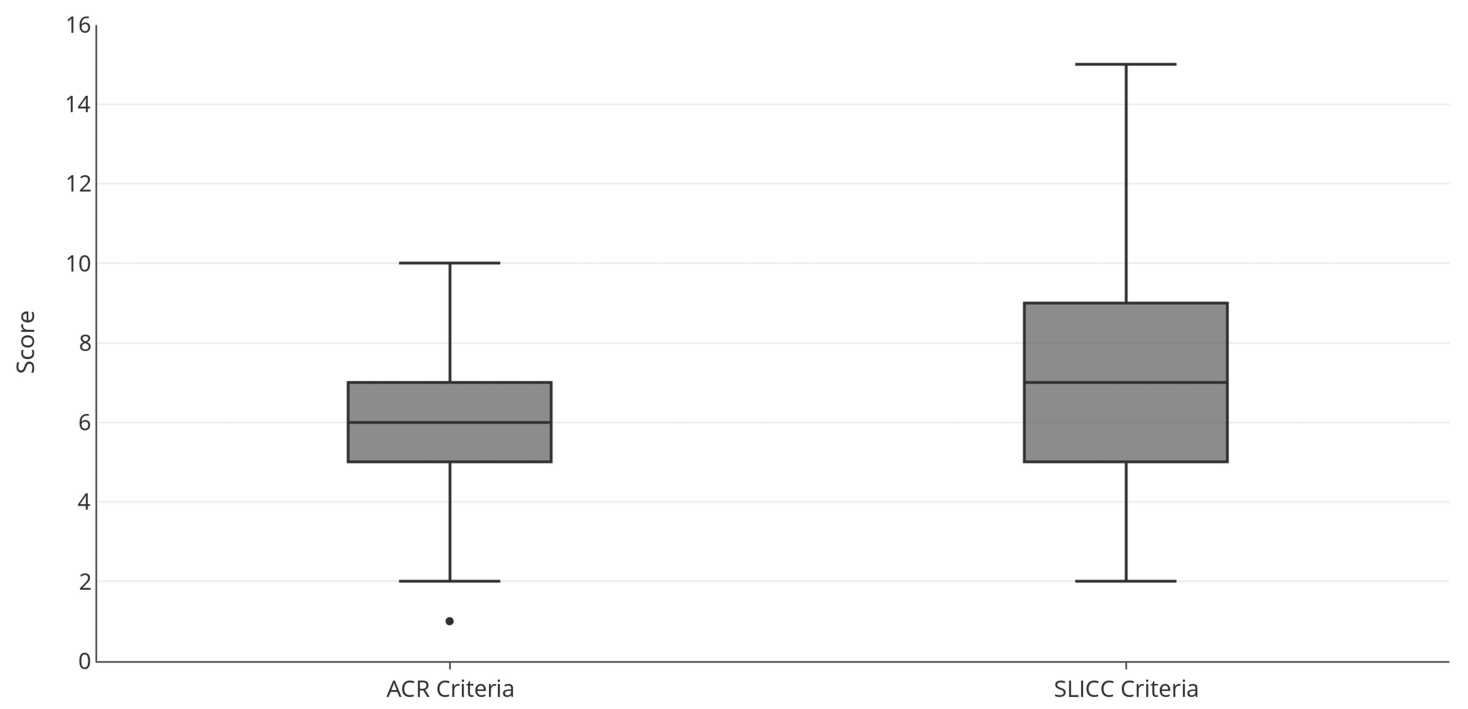

Figure 1. ACR and SLICC score distribution. Box and whisker plot of the number of individual ACR and SLICC criteria met by the cohort. The $\mathrm{X}$-axis shows the classification system, while the Y-axis shows the number of criteria. The box shows the median and interquartile range, while the whiskers show the upper and lower extremes. Dots placed outside the whiskers represent outliers. ACR: American College of Rheumatology; SLICC: Systemic Lupus International Collaborating Clinics.

criteria but $\geq 4$ SLICC classification criteria or confirmed renal biopsy (ACR-/SLICC+; Figure 2A, dark grey circles). (3) Twenty patients $(2.8 \%)$ had $<4$ SLICC classification criteria but $\geq 4$ ACR classification criteria (ACR+/SLICC-; Figure 2A, light grey circles).

(4) Seven patients in the cohort (1\%) did not have $>4$ SLICC or ACR classification criteria and were classified with cSLE by consensus expert opinion only.

$A C R+/ S L I C C+c S L E$ patients. Within the ACR+/SLICC+ population, we examined how the number of classification criteria compared between the 2 classification systems (Table $3)$. Overall, about half the patients $(322 / 647,49.8 \%)$ met

Personal non-commercial use only. The Journal of Rheumatology Copyright @ 2019 . All rights reserved. 
Table 2. Comparison of patients who met 1, both, or neither classification criteria.

\begin{tabular}{|c|c|c|c|c|c|}
\hline Variables & *ACR+/SLICC+ & $* * \mathrm{ACR}+/ \mathrm{SLICC}-$ & ${ }^{ \pm} \mathrm{ACR}-/ \mathrm{SLICC}+$ & ${ }^{ \pm \pm}$ACR-/SLICC- & Total \\
\hline Total & 647 & 20 & 48 & 7 & 722 \\
\hline \multicolumn{6}{|l|}{ Sex } \\
\hline Male & 117 & 2 & 11 & 2 & 132 \\
\hline \multicolumn{6}{|l|}{ Ethnicity } \\
\hline Asian & 247 & 1 & 12 & 4 & 264 \\
\hline Hispanic & 25 & 1 & 2 & 0 & 28 \\
\hline Aboriginal & 4 & 0 & 0 & 0 & 4 \\
\hline Pacific Islander & 1 & 0 & 0 & 0 & 1 \\
\hline Mixed & 36 & 0 & 2 & 0 & 38 \\
\hline Unknown & 54 & 7 & 13 & 1 & 75 \\
\hline \multicolumn{6}{|c|}{ Time of scoring from diagnosis } \\
\hline$>10 \mathrm{yrs}$ & 69 & 0 & 0 & 0 & 69 \\
\hline
\end{tabular}

* Patients who met both ACR and SLICC classification criteria. ** Patients who met ACR but not SLICC classification criteria. ${ }^{ \pm}$Patients who met SLICC but not ACR classification criteria. ${ }^{ \pm \pm}$Patients who met neither ACR nor SLICC classification criteria. ACR: American College of Rheumatology; SLICC: Systemic Lupus International Collaborating Clinics.

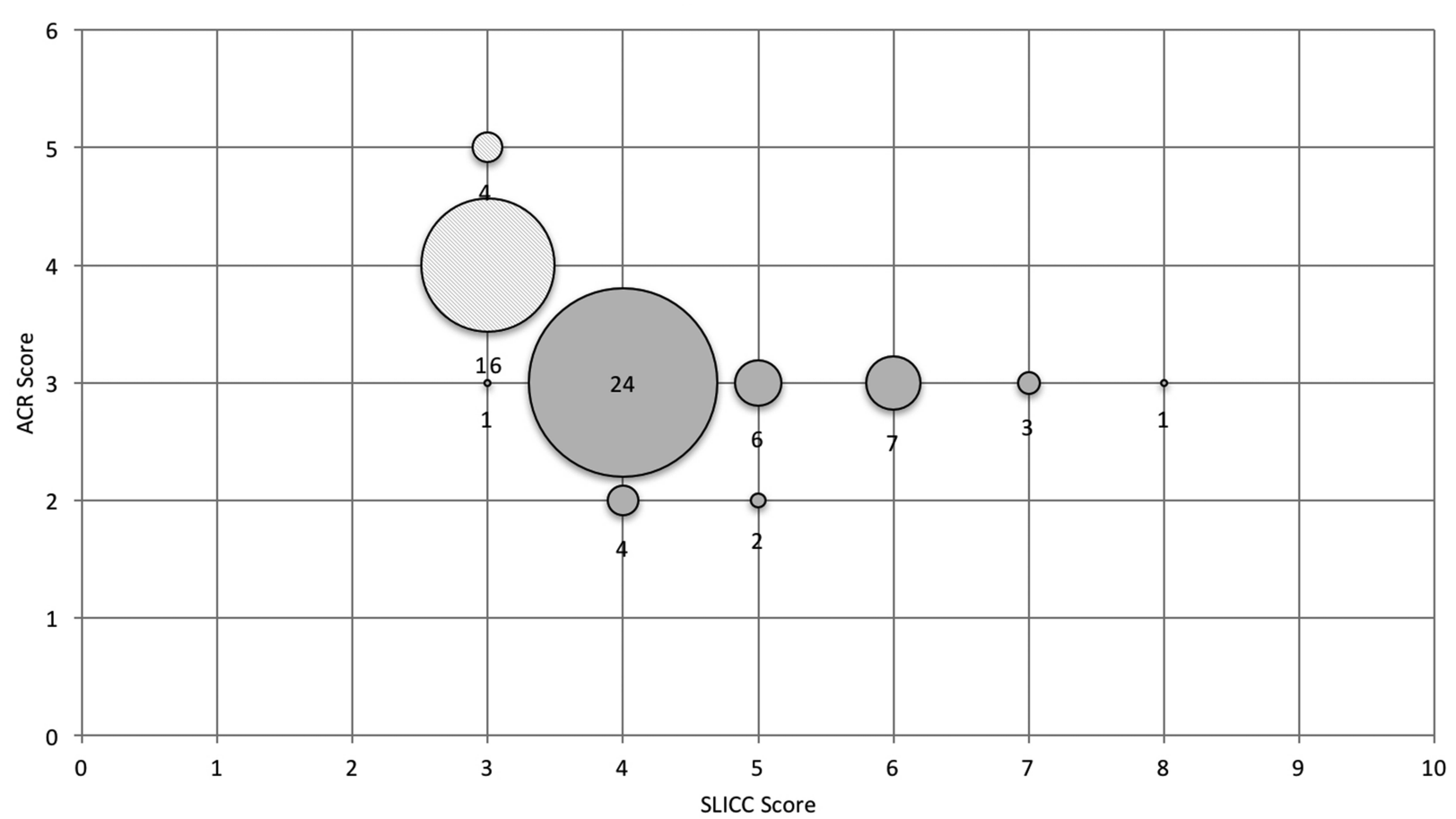

Figure 2A. The individual number of ACR and SLICC criteria met in patients who met only 1 of the 2 classification criteria (ACR-/SLICC+ and ACR+/SLICCpatients). The majority of discordant scores lie only 1 point apart. The location along the X-and Y-axes indicates the number of SLICC and ACR criteria met by each patient, respectively. The diameter of each circle is proportional to the number of cSLE patients (number shown within or below) associated with each locus. The light grey circles represent the $20 \mathrm{ACR}+/ \mathrm{SLICC}-$ patients, the majority (16) of which met 4 ACR criteria and 3 SLICC criteria. The dark grey circles represent the 48 ACR-/SLICC+ patients, the majority (24) of whom met 3 ACR criteria and 4 SLICC criteria. ACR: American College of Rheumatology; SLICC: Systemic Lupus International Collaborating Clinics; cSLE: childhood-onset systemic lupus erythematosus. 
Table 3. Comparison of the number of individual classification criteria met by each ACR+/SLICC+ patient.

\begin{tabular}{lcccc}
\hline $\begin{array}{l}\text { No. ACR } \\
\text { Criteria }\end{array}$ & $\begin{array}{c}\text { ACR } \\
\text { SLICC }\end{array}$ & $\begin{array}{c}\text { ACR }< \\
\text { SLICC** }\end{array}$ & $\begin{array}{c}\text { ACR }> \\
\text { SLICC*** }\end{array}$ & Total \\
\hline 4 & 29 & 63 & $1^{\dagger}$ & 93 \\
5 & 37 & 104 & 18 & 159 \\
6 & 18 & 140 & 12 & 170 \\
$>6$ & 202 & 15 & 8 & 225 \\
Total & 286 & 322 & 39 & 647 \\
\hline
\end{tabular}

$\dagger$ This patient met SLICC classification because of biopsy-confirmed nephritis and autoantibodies. * No. patients who had equal number of ACR and SLICC classification criteria. ** No. patients who had fewer ACR than SLICC classification criteria. *** No. patients who had more ACR than SLICC classification criteria. ACR: American College of Rheumatology; SLICC: Systemic Lupus International Collaborating Clinics.

more SLICC than ACR criteria, a little fewer than half of patients $(286 / 647,44.2 \%)$ had an equal number of ACR and SLICC classification criteria, and only $39(6.0 \%)$ met more ACR than SLICC criteria. One patient was placed in this group despite a SLICC score of 3 because the presence of biopsy-proven nephritis qualified for SLICC classification. For ease of presentation and clinical significance, we designated a maximum score of $>6$ for this analysis.

$A C R-/ S L I C C+c S L E$ patients. Of the 48 patients who were in the ACR-/SLICC+ cohort, 42 had 3 ACR classification criteria and 6 had only 2 . The individual SLICC-specific classification criteria met by these are shown in Table 4 . In the 42 patients who met SLICC criteria but had $3 \mathrm{ACR}$ criteria, 41 met SLICC classification owing to additional SLICC-specific criterion and 1 owing to biopsy-confirmed nephritis. The most common SLICC-specific criterion was hypocomplementemia in 18 , followed by alopecia in 15 , multiple hematologic criteria in 13, multiple specific autoantibodies in 11, positive Coombs test without hemolysis in 6 , broadened criteria of central nervous system (CNS) involvement in 2, and broadened criteria for acute cutaneous lupus erythematosus in 2 (Table 4). Similarly, in the 6 patients who met SLICC criteria but only 2 ACR criteria, 5 patients had both hypocomplementemia and multiple hematologic criteria, while the remaining patient had alopecia and met SLICC (but not ACR) criteria for leukopenia (Table 4). The percentages of the individual SLICC criteria in these patients are as follows: hypocomplementemia (29.1\%), additional hematologic $(24.1 \%)$ criteria, alopecia $(20.3 \%)$, additional immunologic (13.9\%) criteria, positive Coombs test without hemolysis $(7.6 \%)$, broadened criteria for CNS $(2.5 \%)$, and broadened acute cutaneous lupus erythematosus (2.5\%) criteria (Figure 2B).

$A C R+/ S L I C C-c S L E$ patients. All 20 of the patients who were in the ACR+/SLICC - cohort met 3 SLICC classification criteria. The additional ACR classification criteria were photosensitivity in 14, ACR (but not SLICC) lymphopenia classification criteria in 3, and both photosensitivity and ACR (but not SLICC) lymphopenia in 3. Overall, photosensitivity was present in $73.9 \%$ and ACR (but not SLICC) lymphopenia in $26.1 \%$ of the 20 patients (Figure $2 \mathrm{C}$ ).

If we modified SLICC criteria to consider photosensitivity as a separate criterion from acute cutaneous lupus erythematosus rash (as in ACR), the sensitivity of SLICC classification would increase to $98.6 \%$ ( $\mathrm{p}=0.0001)$. The sensitivity would further increase to $99.0 \%(\mathrm{p}=0.0001)$ if both changes were made (disaggregation of photosensitivity and inclusion of the ACR lymphopenia criteria).

Table 4. ACR-/SLICC+ group*, additional SLICC-specific classification criteria.

\begin{tabular}{lccc}
\hline $\begin{array}{l}\text { SLICC Feature Not in ACR } \\
\text { Classification Criteria }\end{array}$ & $\begin{array}{c}{ }^{ \pm} \text {Patients with 3 } \\
\text { ACR (n=41) }\end{array}$ & $\begin{array}{c} \pm \pm \text { Patients with 2 } \\
\text { ACR (n=6) }\end{array}$ & $\begin{array}{c}\text { \% of Individual } \\
\text { SLICC Criteria, n (\%) }\end{array}$ \\
\hline Hypocomplementemia & 18 & 5 & $23 / 79(29.1)$ \\
Alopecia & 15 & 1 & $16 / 79(20.3)$ \\
Total no. with additional hematologic & 13 & 6 & $19 / 79(24.1)$ \\
$\quad$ Leukopenia (SLICC only) & 1 & 1 & \\
$\quad$ additional & 5 & 3 & \\
2 additional & 7 & 2 & $6 / 79(7.6)$ \\
Coombs+ anemia without hemolysis & 6 & 0 & $11 / 79(13.9)$ \\
Total no. with additional autoantibodies & 11 & 0 & \\
$\quad 1$ additional & 9 & 0 & $2 / 79(2.5)$ \\
$\quad 2$ additional & 2 & 0 & $2 / 79(2.5)$ \\
CNS criteria & 2 & 0 & $79 / 79(100)$ \\
Acute cutaneous lupus erythematosus & 2 & 0 & \\
Total & 67 & 12 & \\
\hline
\end{tabular}

* All 48 ACR-/SLICC+ have < 4 ACR classification criteria; 47 met $\geq 4$ SLICC classification criteria, and 1 met SLICC classification through biopsy-confirmed nephritis and the presence of autoantibodies (excluded from this table). ${ }^{ \pm}$Patients could have $>1$ SLICC classification criteria not present in ACR classification. ${ }^{ \pm \pm}$All patients had 2 SLICC classification criteria not present in ACR classification. ACR: American College of Rheumatology; SLICC: Systemic Lupus International Collaborating Clinics; CNS: central nervous system. 


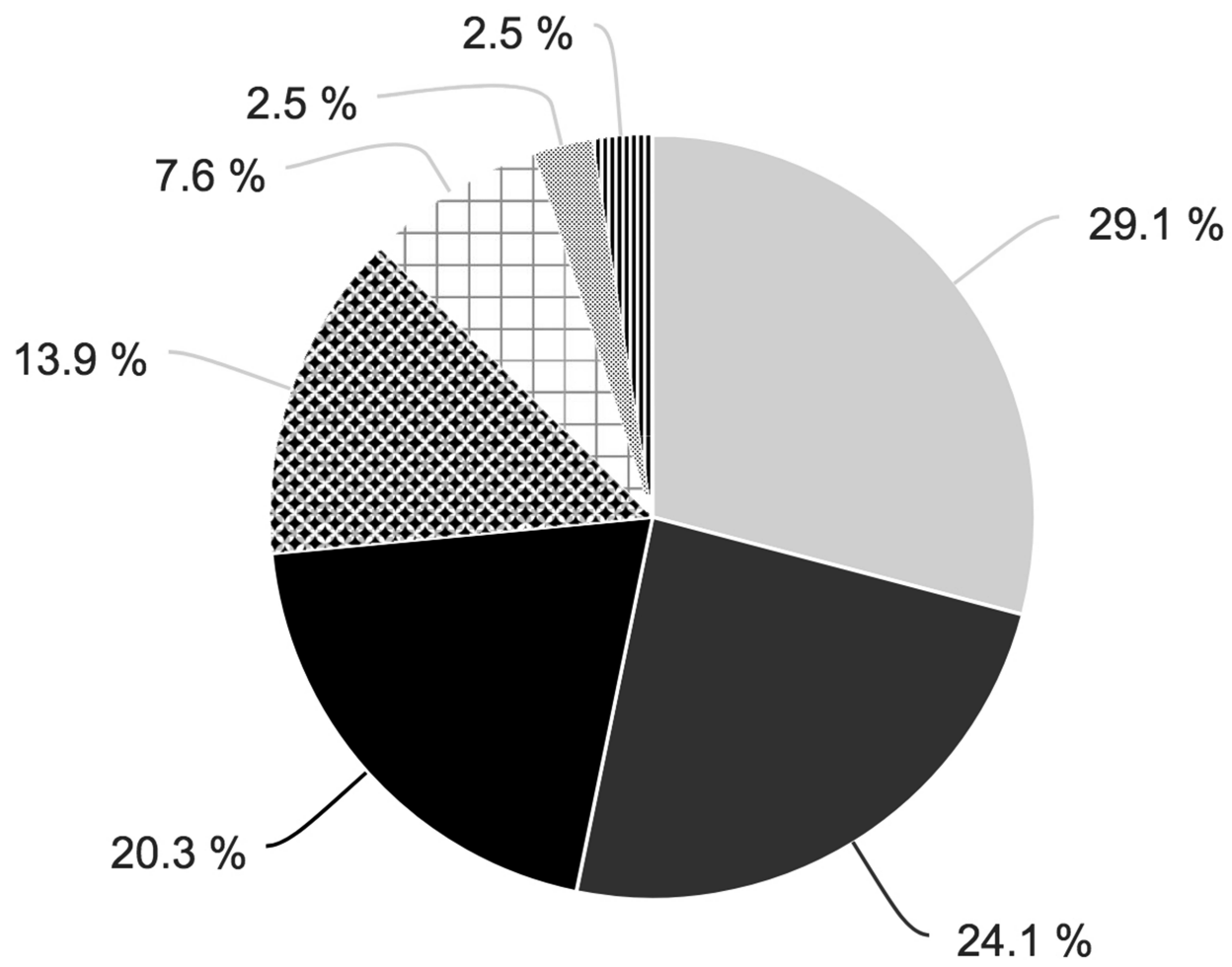

\begin{abstract}
Figure $2 B$. Percentages of the individual classification criteria present in the 48 patients who had $\geq 4$ SLICC criteria but $<4$ ACR criteria (ACR-/SLICC+ patients). This was the result of the following differences between the SLICC and ACR classification criteria: in $29.1 \%$ it was the result of hypocomplementemia (light grey), $24.1 \%$ multiple hematologic criteria (dark grey), 20.3\% alopecia (black), 13.9\% multiple immunologic criteria (diamonds), $7.6 \%$ Coombs+ positive anemia without hemolysis (squares), $2.5 \%$ broadened criteria for CNS criteria (dots), and $2.5 \%$ broadened acute cutaneous lupus erythematosus criteria (stripes). ACR: American College of Rheumatology; SLICC: Systemic Lupus International Collaborating Clinics; CNS: central nervous system.
\end{abstract}

$A C R-/ S L I C C-c S L E$ patients. There were 7 patients in the ACR-/SLICC- cohort. The diagnosis of these patients with cSLE was based on the presence of at least 1 specific SLE-associated autoantibody not included in classification (anti-Ro, -La, or -RNP antibodies) in the absence of anti-dsDNA antibodies (definition was agreed upon by all 3 experts). The majority of these patients (4/7) had anti-Ro antibodies along with 3 ACR and 3 SLICC classification criteria. The remaining 3 patients had anti-Ro and anti-RNP antibodies with 3 ACR and 2 SLICC criteria; anti-Ro and anti-La antibodies with 2 ACR and 2 SLICC criteria; and anti-RNP antibodies with 1 ACR and 2 SLICC criteria, respectively. Anti-Ro antibodies were present in $6(85.7 \%)$ of these patients, anti-RNP antibodies in $2(28.6 \%)$ patients, and anti-La antibodies in $1(14.3 \%)$ patient. None had sicca symptoms or Raynaud phenomenon.

\section{DISCUSSION}

SLE can be difficult to diagnose and classify owing to its heterogeneous clinical presentation. The ACR and SLICC classification criteria were developed to select a well-defined SLE patient population for research purposes. Although both sets of classification criteria have been well validated in adult
SLE populations $\mathrm{s}^{9,10,11,12}$, only a few studies, all with smaller sample sizes, have examined their use in $\mathrm{CSLE}^{6,7,8}$. In our study, we compared the sensitivities of the ACR and SLICC classification criteria in cSLE using a large multiethnic cohort of 722 patients with a clinical diagnosis of cSLE.

The SLICC criteria had significantly increased sensitivity (96.3\%) compared to ACR criteria (92.4\%) in cSLE. This increased sensitivity of the SLICC criteria is similar to what has been reported in both adult ${ }^{5,9,10,11,12}$ and pediatric ${ }^{6,7,8}$ populations. The increased sensitivity of the SLICC criteria remained significant when patients were stratified according to sex. However, when stratified by ethnicity, SLICC criteria was only significantly more sensitive in the Asian subgroup even though a similar trend was also found across other ethnicities.

When we examined how the criteria performed at different times from diagnosis, increased sensitivity of the SLICC criteria was found only within the first 3 years of diagnosis. In later years, the sensitivities of both criteria increased and the difference in sensitivity narrowed between the 2 criteria such that at 10 years, both criteria had 100\% sensitivity. Our findings are similar to the results of other studies, which found ACR and SLICC sensitivities of $84.1 \%$ and $92.9 \%$, 


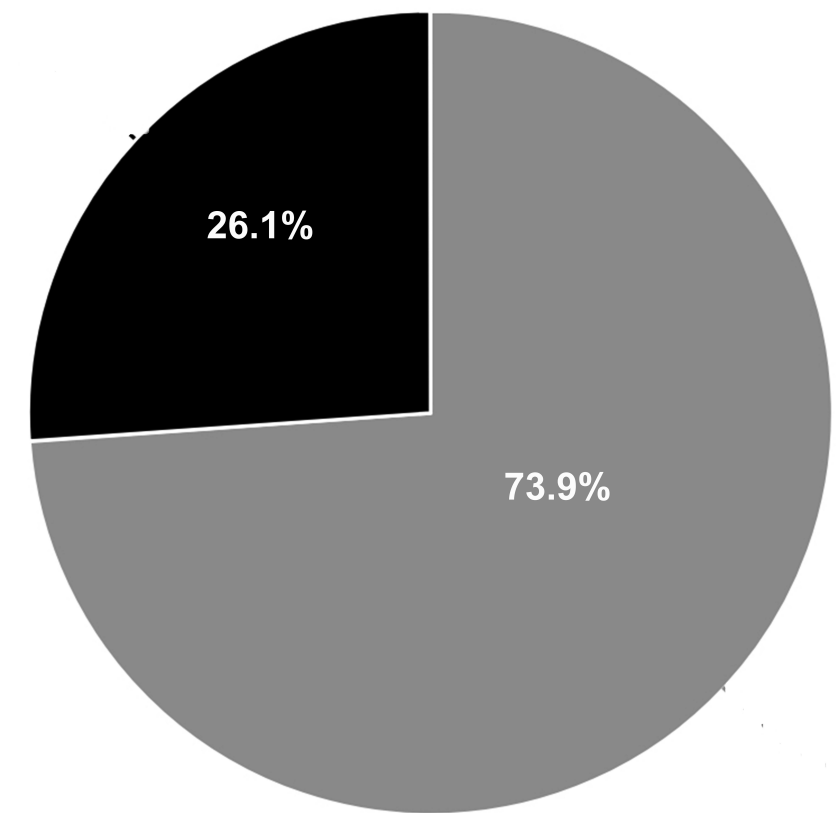

Figure $2 C$. Percentages of the individual classification criteria present in the patients who had $\geq 4$ ACR criteria but $<4$ SLICC criteria (ACR+/SLICCpatients). This was the result of photosensitivity in $73.9 \%$ (grey) and differences in the definition of lymphopenia used in the 2 classification criteria in 26.1\% (black). ACR: American College of Rheumatology; SLICC: Systemic Lupus International Collaborating Clinics.

respectively, within the first year from diagnosis, followed by $92.0 \%$ and $100 \%$ at the latest followup, respectively (median of 3.6 yrs from the initial scoring) ${ }^{8}$. This overarching trend is also supported by the even larger differences in sensitivity reported by Sag, et $a l^{6}$ and Fonseca, et $a l^{7}$ based on scoring at disease onset. Therefore, our study and the other cSLE studies demonstrate that the SLICC classification criteria identifies more patients earlier in their course as compared to the ACR classification criteria. The use of SLICC criteria may improve clinical care and allow us to study patients earlier in their disease course.

Although we found an increased sensitivity of the SLICC criteria in cSLE, as expected, there was excellent concordance at $89.6 \%$ between the 2 criteria to classify a patient with cSLE. This has been previously reported ${ }^{6,7,8}$. When we examined which specific criteria increased the sensitivity of the SLICC criteria as compared to the ACR criteria, we found that the most common criterion was hypocomplementemia. Hypocomplementemia showed a $92.6 \%$ specificity in the derivation cohort for the SLICC criteria validation study ${ }^{5}$. Subsequent studies in adults have confirmed that the addition of hypocomplementemia increased the sensitivity of SLE classification in patients with undifferentiated connective tissue diseases and in patients who did not meet ACR criteria. The number of patients having hypocomplementemia was similar to the number with arthritis and anti-dsDNA antibodies ${ }^{11,12,13}$. We found that not restricting patients to a single hematologic abnormality and the addition of additional autoantibodies (as seen in the SLICC criteria) will lead to an earlier classification of patients with chronic immune-mediated cytopenias including Evans syndrome and potential earlier detection of major organ involvement. Overall, we found that we identified an additional 3.9\% of patients using SLICC criteria.

Conversely, $2.8 \%$ of patients who were classified by ACR criteria would not be classified as having cSLE using the SLICC criteria. In the majority of cases, the loss of photosensitivity as a separate criterion in the SLICC classification criteria led to its failure in classifying a patient that was classified by ACR criteria. In a minority of cases, the loss of SLICC classification was due to the differences in the criteria for lymphopenia. In fact, if we added back photosensitivity as a separate criterion, the SLICC sensitivity would increase to $98.6 \%$ and the use of the ACR and not SLICC lymphopenia criteria would further increase the sensitivity to $99.0 \%$.

Seven patients $(1 \%)$ in our study population were diagnosed with cSLE without meeting either SLICC or ACR criteria. Six of these patients were positive for anti-Ro antibodies (3 of which had no other specific autoantibodies). Anti-Ro antibodies are more frequently seen in patients with primary Sjögren syndrome (pSS) than either adult-onset SLE and cSLE $^{14,15,16,17,18}$. Because pSS occurs in 2-10/10,000 adults ${ }^{19,20}$, the specificity of anti-Ro antibodies in adult-onset SLE is low. In contrast, pSS is very rare in children, with only 81 cases reported in a systematic review of the literature between 2000 and 2010, while it occurs in about $40 \%$ of patients with cSLE ${ }^{21}$. Therefore, although never tested in a pediatric cohort, the specificity of anti-Ro antibodies to cSLE is likely to be high. Although this would affect the classification of cSLE in only a minority of patients, it may be reasonable to consider the utility of including anti-Ro antibodies in the classification criteria in cSLE owing to the rarity of pSS in this age group.

The major limitation of our study is that only sensitivity of the classification criteria was studied, because a lack of an appropriate control group precluded the ability to assess specificity. Similarly, our suggestions regarding the addition of photosensitivity to SLICC criteria, the inclusion of ACR (as opposed to SLICC) lymphopenia criteria, and addition of anti-Ro antibodies would significantly increase sensitivity, but the effect of these changes on specificity must be assessed prior to implementation. In addition, the study was conducted in only a single center, although the SickKids cohort is a multiethnic cohort. Lastly, because there is no gold standard for the diagnosis of SLE, we relied on the consensus clinical diagnosis by expert opinion of 3 clinicians from the same SLE clinic.

The SLICC criteria were significantly more sensitive than ACR criteria in cSLE classification overall and across both sexes in subgroup analysis. Similar but non-statistically significant trends were seen consistently across multiple

Personal non-commercial use only. The Journal of Rheumatology Copyright $\odot$ 2019. All rights reserved. 
ethnicities. SLICC classification criteria was found to be significantly superior to ACR classification criteria early in disease course. With the upcoming European League Against Rheumatism/ACR classification criteria update ${ }^{22}$, it will be interesting to see how it performs in cSLE in comparison.

\section{ACKNOWLEDGMENT}

The authors thank the Canadian Rheumatology Association for a summer scholarship awarded to Jessie Tao.

\section{REFERENCES}

1. Cohen AS, Reynolds WE, Franklin EC, Kulka JP, Ropes MW, Shulman LE, et al. Preliminary criteria for the classification of systemic lupus erythematosus. Bull Rheum Dis 1971;21:643-8.

2. Tan EM, Cohen AS, Fries JF, Masi AT, McShane DJ, Rothfield NF, et al. The 1982 revised criteria for the classification of systemic lupus erythematosus. Arthritis Rheum 1982;25:1271-7.

3. Hochberg MC. Updating the American College of Rheumatology revised criteria for the classification of systemic lupus erythematosus. Arthritis Rheum 1997;40:1725.

4. Petri M, Magder L. Classification criteria for systemic lupus erythematosus: a review. Lupus 2004;13:829-37.

5. Petri M, Orbai AM, Alarcon GS, Gordon C, Merrill JT, Fortin PR, et al. Derivation and validation of the Systemic Lupus International Collaborating Clinics classification criteria for systemic lupus erythematosus. Arthritis Rheum 2012;64:2677-86.

6. Sag E, Tartaglione A, Batu ED, Ravelli A, Khalil SM, Marks SD, et al. Performance of the new SLICC classification criteria in childhood systemic lupus erythematosus: a multicentre study. Clin Exp Rheumatol 2014;32:440-4.

7. Fonseca AR, Gaspar-Elsas MI, Land MG, de Oliveira SK. Comparison between three systems of classification criteria in juvenile systemic lupus erythematosus. Rheumatology 2015; 54:241-7.

8. Lythgoe H, Morgan T, Heaf E, Lloyd O, Al-Abadi E, Armon K, et al; UK JSLE Study Group. Evaluation of the ACR and SLICC classification criteria in juvenile-onset systemic lupus erythematosus: a longitudinal analysis. Lupus 2017;26:1285-90.

9. Amezcua-Guerra LM, Higuera-Ortiz V, Arteaga-Garcia U, Gallegos-Nava S, Hubbe-Tena C. Performance of the 2012 Systemic Lupus International Collaborating Clinics and the 1997 American College of Rheumatology classification criteria for systemic lupus erythematosus in a real-life scenario. Arthritis Care Res 2015;67:437-41

10. Ighe A, Dahlstrom O, Skogh T, Sjowall C. Application of the 2012 Systemic Lupus International Collaborating Clinics classification criteria on a regional Swedish systemic lupus erythematosus register. Arthritis Res Ther 2015;17:3.
11. Ines L, Silva C, Galindo M, Lopez-Longo FJ, Terroso G, Romao VC, et al; Rheumatic Diseases Registry of the Portuguese Society of Rheumatology; Registry of Systemic Lupus Erythematosus Patients of the Spanish Society of Rheumatology. Classification of systemic lupus erythematosus: Systemic Lupus International Collaborating Clinics versus American College of Rheumatology criteria. Arthritis Care Res 2015; 67:1180-5.

12. Bortoluzzi A, Furini F, Campanaro F, Govoni M. Application of SLICC classification criteria in undifferentiated connective tissue disease and evolution in systemic lupus erythematosus: analysis of a large monocentric cohort with a long-term follow-up. Lupus 2017;26:616-22.

13. Ungprasert P, Sagar V, Crowson CS, Amin S, Makol A, Ernste FC, et al. Incidence of systemic lupus erythematosus in a population-based cohort using revised 1997 American College of Rheumatology and the 2012 Systemic Lupus International Collaborating Clinics classification criteria. Lupus 2017;26:240-7.

14. Locht H, Pelck R, Manthorpe R. Clinical manifestations correlated to the prevalence of autoantibodies in a large $(n=321)$ cohort of patients with primary Sjögren's syndrome: a comparison of patients initially diagnosed according to the Copenhagen classification criteria with the American-European consensus criteria. Autoimmun Rev 2005;4:276-81.

15. Song JS, Do JH, Lee SW. The prevalence and the clinical relevance of anti-Ro52 in Korean patients with primary Sjögren's syndrome. Rheumatol Int 2011;32:491-5.

16. Schur PH. Laboratory evaluation of patients with systemic lupus erythematosus. In: Lahita RG, Tsokos G, Buyon JP, Koike T, editors. Systemic lupus erythematosus (5th ed). San Diego: Academic Press; 2011:629-53.

17. Crow MK. Systemic lupus erythematosus. In: Goldman L, Schafer AI, editors. Goldman's cecil medicine (24th ed). Philadelphia: WB Saunders; 2012:1697-705.

18. Silverman E, Eddy A. Systemic lupus erythematosus. In: Cassidy JT, Petty RE, Laxer RM, Lindsley CB, editors. Textbook of pediatric rheumatology (6th ed). Philadelphia: WB Saunders; 2011:315-43

19. Maciel G, Crowson CS, Matteson EL, Cornec D. Prevalence of primary Sjögren's syndrome in a US population-based cohort. Arthritis Care Res 2017;69:1612-6.

20. Goransson L, Haldorsen K, Brun J, Harboe E, Jonsson M, Skarstein $\mathrm{K}$, et al. The point prevalence of clinically relevant primary Sjögren's syndrome in two Norwegian counties. Scand J Rheumatol 2011:40:221-4

21. Patel R, Shahane A. The epidemiology of Sjögren's syndrome. Clin Epidemiol 2014;6:247-55.

22. Aringer M, Dörner T, Leuchten N, Johnson SR. Toward new criteria for systemic lupus erythematosus-a standpoint. Lupus 2016; 25:805-11. 